Research article

\title{
Simultaneous intra/extravascular administration of antiproliferative agents as a new strategy to inhibit restenosis: The peak of reactive cell proliferation as a hallmark for the duration of the treatment Rainer Voisard*, Eva Kucharczyk, Ute Deininger, Regine Baur and Vinzenz Hombach
}

\author{
Address: Department of Internal Medicine II - Cardiology ( Voisard, M.D., Eva Kucharczyk, Ute Deininger, Regine Baur, Vinzenz Hombach, M.D.), \\ University of Ulm, Germany \\ E-mail: Rainer Voisard* - rainer.voisard@medizin.uni-ulm.de; Eva Kucharczyk - eva.kucharczyk@medizin.uni-ulm.de; \\ Ute Deininger - ute.deininger@medizin.uni-ulm.de; Regine Baur - regine.baur@medizin.uni-ulm.de; \\ Vinzenz Hombach - vinzenz.hombach@medizin.uni-ulm.de \\ ${ }^{*}$ Corresponding author
}

Published: 18 January 2002

Received: 9 May 2001

BMC Cardiovascular Disorders 2002, 2:2

Accepted: 18 January 2002

This article is available from: http://www.biomedcentral.com/|47I-226I/2/2

(c) 2002 Voisard et al; licensee BioMed Central Ltd. Verbatim copying and redistribution of this article are permitted in any medium for any purpose, provided this notice is preserved along with the article's original URL.

\begin{abstract}
Background: Strictly intravascular approaches for the treatment of postangioplasty restenosis are effective in the intima and the inner parts of the media but may be insufficient to control redundant pathways in the more outer parts of the media and the adventitia. An inverse situation may occur subsequently to a strictly extravascular approach, like the recently suggested pericardial approach in pigs. We hypothesized that simultaneous intra/extravascular administration of anti-restenotic agents inhibits restenosis by blocking all stimulatory pathways in the entire arterial wall.
\end{abstract}

Methods: Fresh hearts of 25 domestic pigs were obtained from a local slaughterhouse. Left anterior descending coronary arteries (LAD) were harvested, cut into cylindric $5 \mathrm{~mm}$ segments, and cultured as ex vivo porcine organ cultures (POCs). After 9 bar ballooning simultaneous intra/ extravascular administration of high dose diltiazem $(50 \mu \mathrm{g} / \mathrm{mL})$ was carried out for a period of $\mathrm{I}, 2$, 3, 4, 5, 6, and 7 days. At day 7 and 28 proliferative activity (BrdU), neointimal thickening, and staining against smooth muscle $\alpha$-actin and vWF was analysed.

Results: 7 days after ballooning administration of diltiazem for 4, 5, 6, and 7 days inhibited reactive cell proliferation by more than $50 \%$ (n.s.) as compared to control, 28 days after ballooning administration for 6 and 7 days inhibited neointimal thickening by more than 75\% $(p<0.05)$. Simultaneous intra/extravascular administration of high dose diltiazem did not affect the expression of VWF in endothelial cells or smooth muscle $\alpha$-actin in smooth muscle cells.

Conclusions: Simultaneous intra/extravascular administration of high dose diltiazem $(50 \mu \mathrm{g} / \mathrm{mL})$ has to be maintained for at least 6 days to achieve a significant inhibition of neointimal thickening. The data demonstrate the importance of the maximal reactive cell proliferation $(=$ day 7 in the POC-model) for the calculation of the duration of the treatment period. 


\section{Introduction}

Despite the outstanding success of coronary angioplasty and related techniques the problem of restenosis [1] has not yet been overcome. This is predominantly caused by the unexpected complexity of the reaction of the coronary artery wall to a relatively simple aggression with an intravascular device. In a very schematic way the main events after coronary artery ballooning are dissection, elastic recoil, thrombosis, leukocyte attack, reactive cell proliferation, and arterial remodeling [2]. Among these reactive cell proliferation remains extremely difficult to control, although many therapeutical agents have a strong antiproliferative effect [3]. This may be due to the fact that after vascular injury a huge amount of signal transduction cascades is activated in the different cell types within the entire arterial wall.

Intravascular anti-restenotic drug regimens are predominantly effective in the inner layers of the artery wall, upregulation of redundant pathways in the outer parts of the media and the adventia are not or not entirely inhibited. Therefore we hypothesized that simultaneous intra/extravascular administration of antiproliferative agents inhibits restenosis by blocking all redundant pathways [4]. In vivo simultaneous intra/extravascular administration could be carried out by a combination of drug-eluting stents with the pericardial approach [5-7].

The domestic pig is the experimental in vivo model that mimics the characteristic features of human restenosis most closely [8]. In order to limit the number of obligatory experimental studies we have suggested previously to employ organ cultures of porcine coronary arteries (POCs) that had been obtained from a local slaugtherhouse [9]. Due to the fact that internal and external surfaces of the coronary artery segments in the POC-model have full contact with the culture medium, simultaneous intra/ extravascular drug therapy can be mimiced easily by adding a potential anti-restenotic agent to the culture medium [4].

In a cascade of various prescreening and complex in vitro models of human restenosis [10] we have recently demonstrated a significant antiproliferative effect of the calcium channel blocker diltiazem in a concentration of $50 \mu \mathrm{g} /$ $\mathrm{mL}$, a toxic effect could be excluded. Due to the fact that this very high concentration is settled two orders of magnitudes above the maximal plasma level subsequent to systemic administration, it can be achieved barely in a local high dose approach. In the POC-model the maximal reactive cell proliferation after ex vivo ballooning occurres at day 7 , followed by a maximum of neointimal thickening at day 28 [9]. In the current study we added high dose diltiazem for 1, 2, 3, 4, 5, 6, and 7 days to the POC-model and studied, howmany days the antiproliferative regime has to be maintained in order to accomplish a significant inhibition of neointimal thickening at day 28 .

\section{Methods}

\section{Preparation of coronary organ cultures}

Fresh hearts of 25 pigs (age 3-5 months, weighing 100$120 \mathrm{~kg}$ ) were obtained from a local slaughterhouse. Left anterior descending coronary arteries (LAD) were prepared and removed carefully from the epicardial surface. Cylindric segments with a side lenght of $5 \mathrm{~mm}$ were cut from the LAD-segments. Immediately after preparation, LAD-cylinders were transfered as POC into sterile HEPESbuffered $(15 \mathrm{mmol} / \mathrm{L})$ culture medium (DMEM) without serum supplement.

\section{Ex vivo ballooning}

For ex vivo angioplasty POCs were incubated with a standard $3 \mathrm{~mm}$ balloon catheter (Medtronic 14K2030E, Diisseldorf, Germany) and treated with 9 bar for $60 \mathrm{~s}$, the balloon/artery ratio was 1 . If tearing occurred in the artery wall, the segments were not used for the study. The inflation of balloon catheters was carried out with Aqua bidest.

\section{Simultaneous intralextravascular application of high dose diltiazem}

After ex vivo ballooning high dose diltiazem (Gödecke, Freiburg im Breisgau, Germany) was added to the culture medium in a concentration of $50 \mu \mathrm{g} / \mathrm{mL}$. The agent was dissolved in distilled water. Simultaneous intra/extravascular application of high dose diltiazem was carried out by incubation of POC with diltiazem for a period of 1,2 , $3,4,5,6$, and 7 days. At each medium exchange the drug was renewed.

\section{Cultivation and fixation of organ cultures}

Immediately after in vitro ballooning LAD-segments were transfered to 6 well plates (Tecnomara, Fernwald, Germany) and cultured in a mixture of Waymouth's MB 752/1 and Ham F12 nutrient mixture $(1: 1 \mathrm{v} / \mathrm{v}$; Bio Whittaker, Verviers, Belgium) supplemented with $15 \%$ fetal calf serum (Bio Whittaker, Verviers, Belgium) at $37^{\circ} \mathrm{C}$ in $5 \%$ $\mathrm{CO}_{2}$. The culture medium was exchangedevery second or third day or if diltiazem had to be administered. Culture conditions for the control groups (angioplasty without diltiazem and neighter angioplasty nor diltiazem) were exactly thesame as described for the angioplasty/diltiazem group.

\section{Analysis of postangioplasty reactive cell proliferation}

Paraffin sections were processed for immunohistological staining using the avidin-biotin immunoperoxidase technique [9]. Bromodeoxyuridine (BrdU), a thymidine analogue, is incorporated into the DNA of S-phase proliferating cells. $18 \mathrm{~h}$ foregoing fixation of the LAD organ cultures BrdU (20 $\mu \mathrm{M}$, Serva, Heidelberg, Germany) 
was added to the culture media supplemented with $20 \mu \mathrm{M}$ deoxycytidine (Serva, Heidelberg, Germany) in order to determine the extent of the cells undergoing DNA synthesis, as described [9]. As secondary antibody a biotinylated horse-anti-mouse antibody (Camon, Wiesbaden, Germany) was used. The number of proliferating cells in the media and in the neointima was calculated independendly as: Number of BrdU-positive cells/Total cell number $\times$ 100. Only sections cut perpendicular to the vessel wall axis were used for the analysis, five sections from different LAD segments were evaluated microscopically in each single experiment at a magnification of $\times 40$.

\section{Identification of smooth muscle cells}

Smooth muscle cells (SMC) were identified by positive staining of sections with antibodies against smooth muscle $\alpha$-actin (Renner, Darmstadt, Germany) at a concentration of $1 \mu \mathrm{g} / \mathrm{mL}$. The detection of the primary antibody was carried out in thesame way as described for the BrdU antibody (see above).

\section{Identification of endothelial cells}

Endothelial cells (EC) in sections of organ cultures were identified by positive staining with antibodies against von Willebrand factor (vWF, INCSTAR, Stillwater, USA). The detection of the primary antibody was carried out in thesame way as described for the BrdU antibody (see above).

\section{Morphometric analysis of neointimal hyperplasia}

For histological examination deparaffinized sections were stained with a modified elastica-van Gieson staining (Chroma, Köngen, Germany). For calculation of the average arterial wall thickening after ex vivo ballooning five cuts from different organ culture segments were analyzed three times for each single experiment with a computerized digital image analyzer (Bioquant TM System $4 \mathrm{R}+\mathrm{M}$ Biometrics, Bilancy Consulting, Düsseldorf, Germany). Taking into account the various lumina of POC segments, the percentage of neointimal thickening was calculated as: Area of neointima/Area of neointima and media $\times 100$.

\section{Statistical analysis}

Data are presented as mean \pm S.D. The two-tailed U-test of Mann, and Whitney was used for determining statistical significance of differences. Statistical significance was accepted for $P<0.05$ [11].

\section{Results}

Effect of simultaneous intralextravascular administration of high dose diltiazem on reactive postangioplasty cell proliferation

The effect of simultaneous intra/extravascular administration of high dose diltiazem on relative reactive cell proliferation both in the neointima and the media of POC segments was studied 7 and 28 days after ex vivo ballooning.

Seven days after ex vivo ballooning in the neointima a 39.4 fold increase of proliferative activity (n.s.) was detected, the number of BrdU-positive cells was increased from $0.1 \pm 0.34 \%$ in untreated controls to $4.3 \pm 5.6 \%$ (Fig. 1 and 2). After simultaneous intra/extravascular administration of diltiazem for 1 and 2 days the number of BrdUpositive cells was $2.8 \pm 6.1 \%$ and $1.1 \pm 1.8 \%$ corresponding to an inhibition by 36 respectively $75 \%$. Administration of diltiazem for 3 days resulted in an increase of BrdU-positive cells by 52\%, 6.6 $\pm 10.4 \%$ of BrdU-positive cells were detected. After incubation of POC-segments with diltiazem for $4,5,6$, and 7 days the percentage of BrdU-positive cells was $0.8 \pm 1.1 \%, 1.9 \pm 3.6 \%, 0.3 \pm$ $0.7 \%$, and $1.2 \pm 1.8 \%$ corresponding to an inhibition by $81 \%, 57 \%, 93 \%$, and $72 \% .28$ days after ballooning the number BrdU-positive cells was very small, $0.0 \pm 0.0 \%$ of BrdU-positive cells were detected in untreated controls and in the POC balloon-group. Incubation with diltiazem for 1,2 , and 3 days resulted in $0.0 \pm 0.0 \%, 1.2 \pm 2.6 \%$, and $0.1 \pm 0.2 \%$ of BrdU-positive cells, respectively. 28 days after incubation with diltiazem for $4,5,6$, and 7 days the number of BrdU-positive cells was $2.2 \pm 5.9 \%, 3.2 \pm 7.2 \%$, $0.0 \pm 0.0 \%$, and $1.1 \pm 2.4 \%$.

In the media the number of BrdU-positive cells was always very low, no matter whether or not cells were incubated with diltiazem or treated with the balloon or both. 7 days after ballooning $0.0 \pm 0.0 \%$ of BrdU-positive cells were detected in untreated controls, $0.2 \pm 0.2$ after 9 bar ballooning. Incubation with diltiazem for 1, 2, and 3 days resulted in $0.1 \pm 0.0 \%, 0.0 \pm 0.0 \%$, and $0.7 \pm 1.3 \%$ of BrdU-positive cells. After incubation with diltiazem for 4, 5,6 , and 7 days the number of BrdU-positive cells was 0.0 $\pm 0.0 \%, 0.1 \pm 0.2 \%, 0.1 \pm 0.1 \%$, and $0.0 \pm 0.0 \%$. 28 days after ballooning $0.0 \pm 0.0 \%$ were found in untreated controls and after 9-bar ballooning. Incubation of POC-segments with diltiazem for 1,2 , and 3 days resulted in $0.1 \pm$ $0.1 \%, 0.1 \pm 0.2 \%$, and $0.0 \pm 0.0 \%$ of BrdU-positive cells, after incubation for $4,5,6$, and 7 days $0.1 \pm 1.4 \%, 0.1 \pm$ $0.3 \%, 0.1 \pm 0.1 \%$, and $0.2 \pm 0.4 \%$.

No statistical significance was reached seven and 28 days after ballooning between the number of BrdU-positive cells in the balloon-group in comparison to the balloon/ diltiazem group.

Effect of simultaneous intralextravascular administration of high dose diltiazem on neointimal thickening

The effect of simultaneous intra/extravascular administration of high dose diltiazem on relative neointimal thickening was analysed 7 and 28 days after 9 bar ballooning (Fig. 3 and 4). 

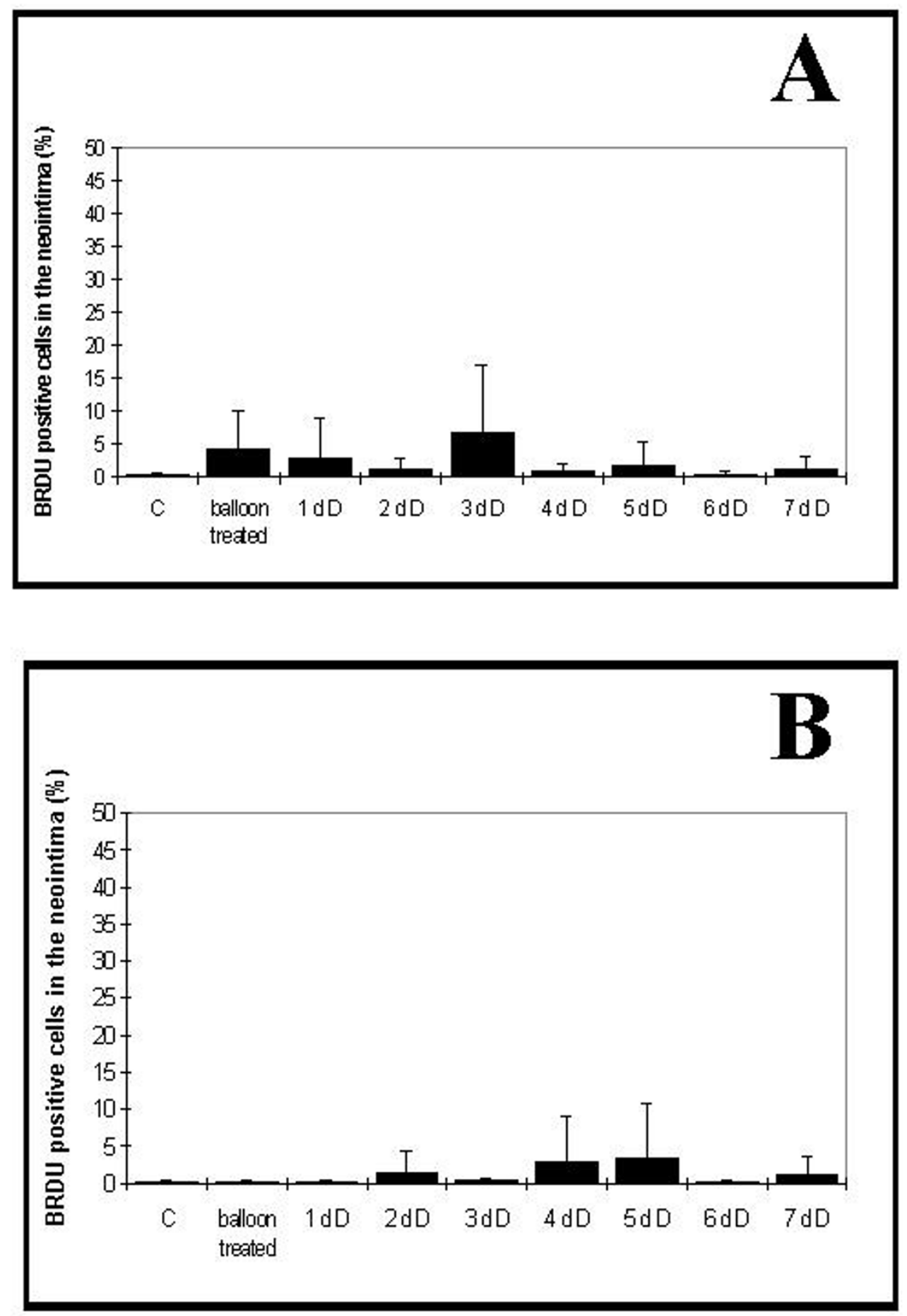

Figure I

Effect of simultaneous intra/extravascular administration of high dose diltiazem $(50 \mu g / \mathrm{mL})$ for I -7 days on time course of cells undergoing DNA synthesis in coronary organ cultures 7 (A) and 28 days (B) after ex vivo ballooning. The number of cells in the neointima with positive bromodeoxyuridine incorporation is depicted in relation to the total cell number. 


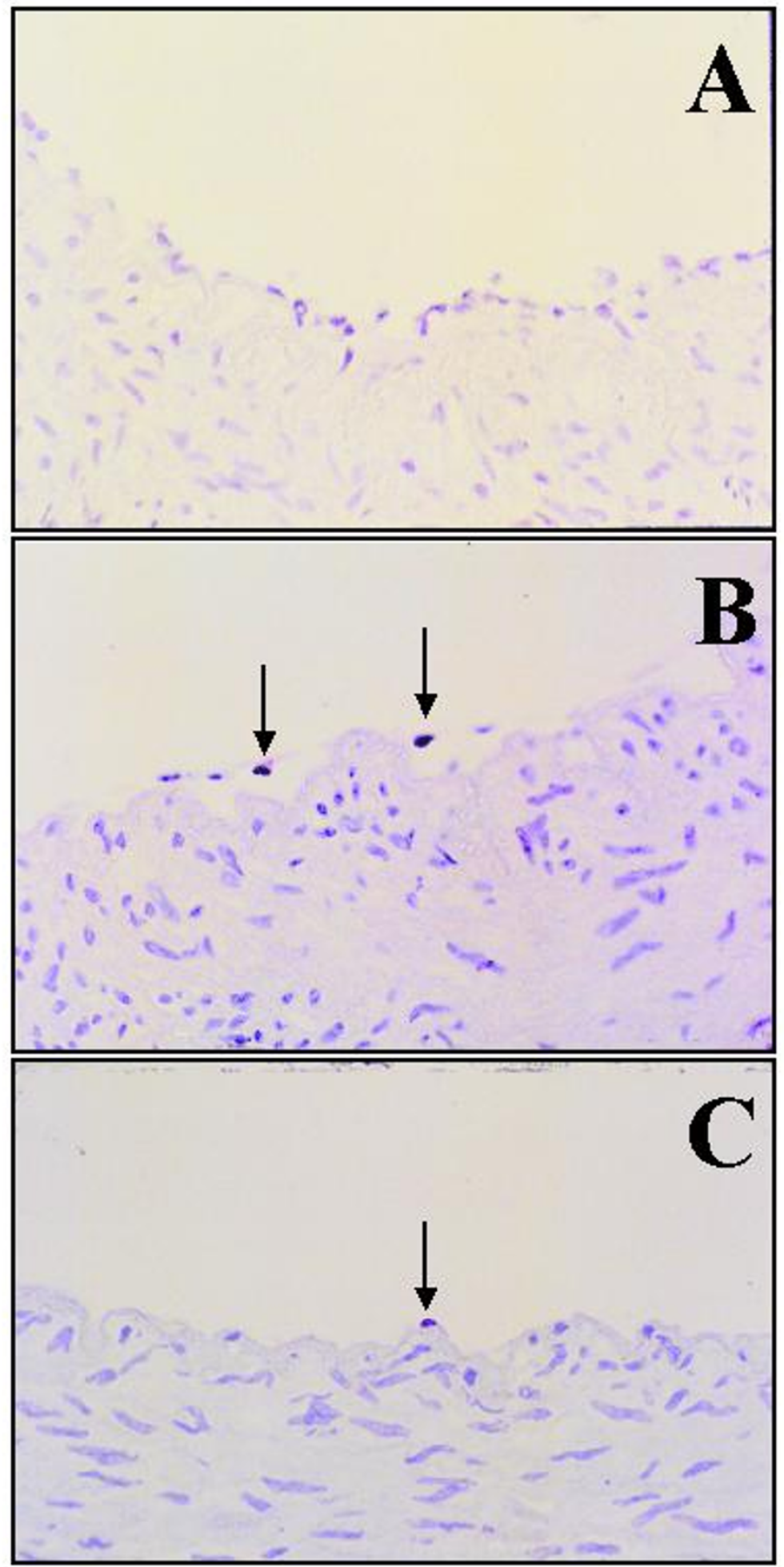

Figure 2

Micrographs showing serial sections of porcine coronary organ cultures at day 7, staining with BrdU (avidin /biotin method): untreated control $(A)$, ex vivo ballooning (B)and ex vivo ballooning with simultaneous intra/extravascular antiproliferative drug therapy $(C)$. arrows = cells with positive staining against BrdU. 

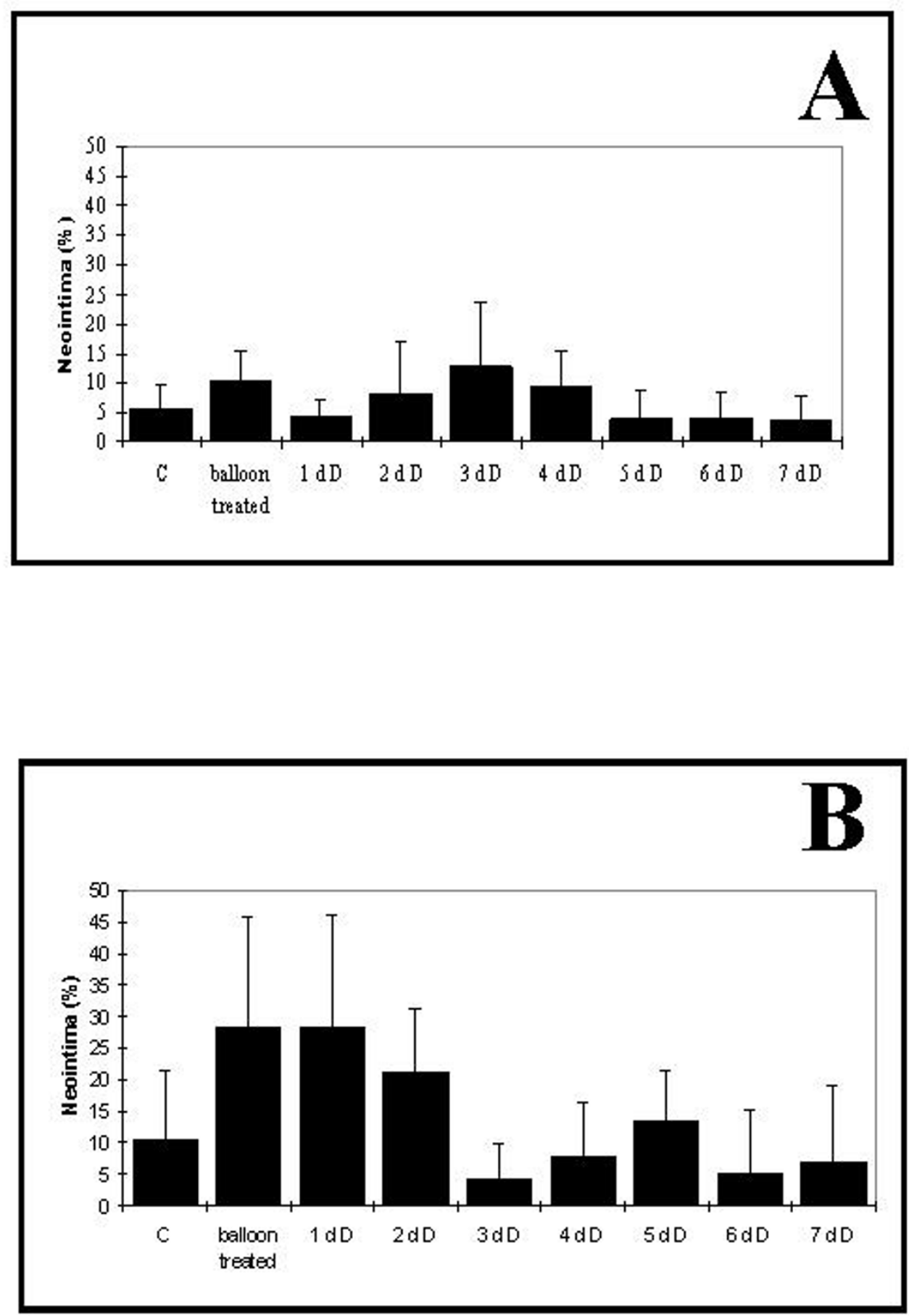

Figure 3

Effect of simultaneous intra/extravascular administration administration of high dose diltiazem $(50 \mu \mathrm{g} / \mathrm{mL})$ for I - 7 days on neointimal thickening in porcine organ cultures $7(A)$ and 28 days (B) after ballooning. Each column represents five different vessels, ${ }^{*}=p<0.05$. 

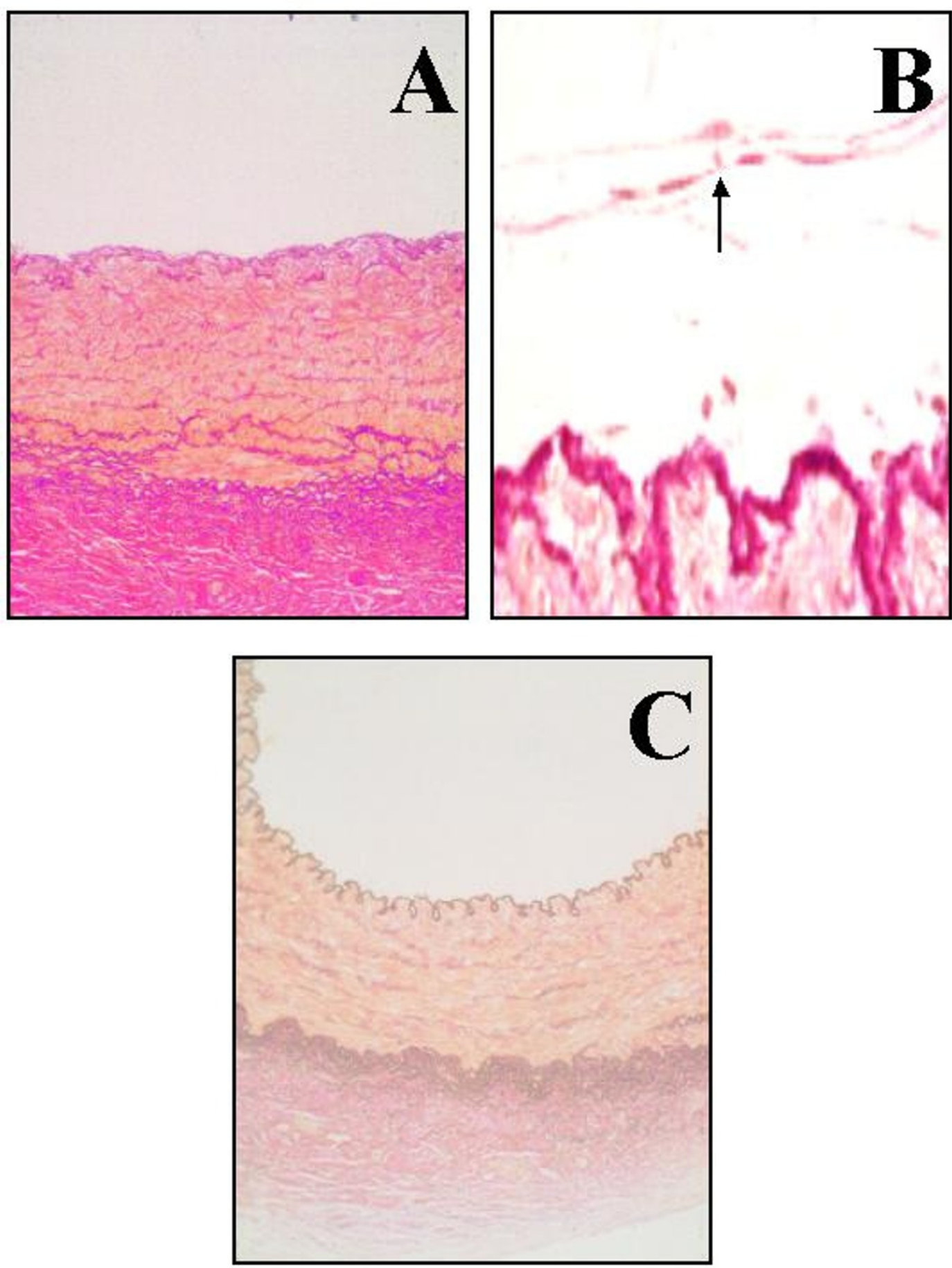

Figure 4

Micrographs of sections of porcine coronoary organ cultures at day 28, staining with elastica van gieson: untreated control (A), ex vivo ballooning $(B)$ and ex vivo ballooning with simultaneous intra/extravascular antiproliferative drug therapy $(\mathrm{C})$. arrow $=$ area of neointimal thickening. 
7 days after ballooning neointimal thickening in the balloon treated group was significantly increased 1.8 fold ( $\mathrm{p}$ $<0.05$ ) from $5.5 \pm 4.0 \%$ in the untreated group to $10.3 \pm$ $4.8 \%$. simultaneous intra/extravascular incubation with diltiazem for 1 and 2 days caused an inhibition of neointimal thickening by 59\% (n.s.) and 21\% (n.s.), corresponding to $4.2 \pm 3.0 \%$ and $8.1 \pm 8.8 \%$. After simultaneous intra/extravascular administration of diltiazem for 3 days

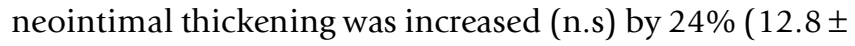
$11 \%)$. Incubation of POC-segments for 4, 5, 6, and 7 days resulted in a neointimal thickening of $9.1 \pm 6.3 \%, 4.0 \pm$ $5.0 \%, 4.0 \pm 4.5 \%$, and $3.7 \pm 4.1 \%$, corresponding to an inhibition (n.s.) by $12 \%, 61 \%, 61 \%$, and $64 \%$.

28 days after ballooning neointimal thickening of POC segments was significantly ( $\mathrm{p}<0.05)$ increased 2.6 fold from $10.5 \pm 11.0 \%$ in untreated controls to $28.4 \pm 17.8 \%$. No effect was found after incubation with diltiazem for 1 day. Incubation with diltiazem for 2 and 3 days resulted in an inhibition of neointimal thickening by $25 \%$ (n.s.) and $86 \%$ ( $\mathrm{p}<0.05)$, corresponding to $21.2 \pm 10.0 \%$ and $4.1 \pm 5.9 \%$. After Incubation of POC-segments for $4,5,6$, and 7 days with diltiazem neointimal thickening was inhibited by $73 \%, 53 \%, 82 \%(\mathrm{p}<0.05)$ and $76 \%(\mathrm{p}<0.05)$, corresponding to $7.7 \pm 8.8 \%, 13.3 \pm 8.0 \%, 5.1 \pm 10.2 \%$, $6.8 \pm 12.1 \%$.

\section{Identification of smooth muscle and endothelial cells}

In the media of POC segments staining with antibodies against smooth muscle $\alpha$-actin was always positive, no matter whether or not organ cultures were treated with ballooning or diltiazem or both. The number of smooth muscle $\alpha$-actin positive cells in the media decreased with increased time in culture, only few POC-segements stained positive after 28 days in culture. In the neointima of POC segments staining against smooth muscle $\alpha$-actin was negative after ballooning and negative after ballooning and EP diltiazem incubation.

Staining of POC-segments with antibodies against vWF was always positive in untreated controls, but decreased after ballooning, simultaneous intra/extravascular incubation with high dose diltiazem did not further decrease the number of cells with positive staining against vWF.

\section{Discussion}

Simultaneous intra/extravascular administration of antiproliferative agents is in a way the maximal strategy to inhibit all pathways that might have been activated after angioplasty. This ex vivo study demonstrates two major findings: 1) the POC-model is a suitable model to study this maximal antiproliferative strategy, and 2) the simultaneous intra/extravascular antiproliferative approach has to be maintained for 6 or 7 days, if a significant inhibition of neointimal thickening shall be achieved. This period of time is almost identical with the peak of reactive cell proliferation in the POC-model [9].

Activation of SMC proliferation and migration after angioplasty via abundant stimulatory pathways is not restrained to inner layers of the vessel wall. Recently Ciezki et al. [12] have reviewed autopsy records of patients who received PTCA prior to death. The authors located sites of cellular proliferation by immunohistochemical staining and demonstrated that reactive cell proliferation after PTCA was found throughout the treated arterial section's axial plane. An anti-restenotic approach that is administered selectively from the intravascular surface of a dilated artery will inhibit mainly stimulatory pathways that activate SMC proliferation and migration in the inner layers of the artery wall but will fail to inhibit stimulatory pathways in the outer parts of the media and in the adventitia. This may explain why local antiproliferative strategies that are administered via selective endoluminal delivery systems as e.g. the Scimed Dispatch Coronary Infusion Catheter failed to inhibit experimental restenosis [13].

A new dimension of antiproliferative drug therapy has been opened by suggestions to use the pericardium as an perivascular drug depot [5-7]. Verrier reported of a successful transatrial access to the normal pericardial space in 6 dogs and 13 pigs [5], Laham et al. [6] demonstrated in 49 yorkshire pigs a safe subxyphoid access. First clinical experiences with the subxiphoid access have been reported lately by Macris et al. [7]. Although the pericardial approach is a very encouraging technique that will acquire its place in the treatment of restenosis within the next years it should be kept in mind that a strictly extravascular approach may produce the inverse situation that stimulatory pathways of reactive cell proliferation are inhibited in the adventia and the more distal parts of the media but not in the neointima and the inner parts of the media. The simultaneous intra/extravascular administration of antiproliferative agents is likely to inhibit consequently all stimulatory pathways in the entire injured artery wall.

Coronary arteries of domestic pigs respond in a similar way to vascular injury as do human coronary arteries [8]. In order to reduce the number of indispensable animal studies we characterized in a previous study a model of restenosis with coronary organ cultures of domestic pigs obtained from a local slaughterhouse [9]. In these POCs the maximal reactive proliferation of SMC occurred at day 7 after ex vivo ballooning, the maximal neointimal thickening was detected at day 28. By adding of antiproliferative agents to the culture medium POCs progress to an elegant ex vivo model for studies of simultaneous intra/ extravascular drug administration [4], because both intravascular and extravascular surfaces of the $5 \mathrm{~mm}$ cylindric 
organ culture segments have full contact with the agents administered to the culture medium.

In POCs reactive cell proliferation after ex vivo ballooning was reduced by more than $50 \%$ after simultaneous intra/ extravascular administration of diltiazem for 4, 5, 6 or 7 days (n.s.), neointimal thickening was significantly inhibited after high dose diltiazem incubation for 6 or 7 days. A strong inhibitory effect of diltiazem is in accordance with prior reports of our laboratory that diltiazem in a concentration of $50 \mu \mathrm{g} / \mathrm{mL}$ significantly inhibited reactive cell migration and proliferation in various in vitro models of human coronary restenosis [10]. Clinical studies applying diltiazem as anti-restenotic agent after peroral administration have not been successful [14,15], with the exception of one study [16] that has only been reported as an abstract. Peak plasma levels of diltiazem are approximately $0.12 \mu \mathrm{g} / \mathrm{mL}$ after a $90 \mathrm{mg}$ dose and $0.15 \mu \mathrm{g} / \mathrm{mL}$ after a $120 \mathrm{mg} /$ dose [17], the administered dosage of diltiazem in these clinical studies varied between $90 \mathrm{mg}$ twice a day [16] and $120 \mathrm{mg}$ three times a day [15]. The negative results of systemically administered diltiazem in these clinical studies was not surprising, because plasma levels were settled more than two orders of magnitudes below the concentrations required for a significant antiproliferative effect [10].

In accordance with prior reports of Koo and Gotlieb [18] and our laboratory [9] staining against smooth muscle $\alpha$ actin in the media was constantly clearly positive. Simultaneous intra/extravascular incubation of POCs with diltiazem in a concentration of $50 \mu \mathrm{g} / \mathrm{mL}$ did not convert the $\alpha$-actin structures of SMC in the media. These data are supported by the fact that diltiazem in concentration of $50 \mu \mathrm{g} / \mathrm{mL}$ did not alter the cytoskeletal structures of smooth muscle $\alpha$-actin, vimentin, and $\alpha$-tubulin structures in human coronary SMC in vitro [10]. Staining against smooth $\alpha$-actin in the neointima was negative at day 7 and day 28. Kocher et al. [19] have suggested that one possible explanation for this phenomenon may be that actively proliferating SMC express a different F-actin phenotype from the $\alpha$-isoform recognized by the smooth muscle $\alpha$-actin antibody. This explanation is confirmed by the fact that reactive cell proliferation in the POC was very low in the media but increased in the neointima.

Staining of POC-segments with antibodies against vWF was always positive in untreated controls, but decreased after ballooning. This result is in accordance with the hypothesis that endothelial injury is an important stimulus for reactive cell proliferation after angioplasty. EC did not recover after beeing damaged during the ballooning, the number of vWF factor positive cells was identical 7 and 28 days after ballooning. The missing recovery of EC in POCs was probably predominantly caused by the fact that we did not use EC-specific culture medium. Simultaneous intra/extravascular administration of high dose diltiazem for up to seven days did not further decrease the number of vWF positive cells. This may be substantial for a sufficient re-endotheliazation of the inner vessel wall after angioplasty in vivo.

The uncertain correlation between data of in vitro and in vivo studies on one side and animal and human species on the other side is a fundamental problem of restenosis research. We have recently demonstrated in $3 \mathrm{D}$ human coronary in vitro model [20] that the positive effects of aspirin in early atherosclerosis and restenosis can only be achieved if a concentration of $5 \mathrm{mM}$ is administered, more than 10-times the commonly administered oral dosage. Although the ex vivo POC-model is very close to the in vivo situation in the domestic pig [21], potential problems may arise by the absence of blood flow, blood pressure and shear stress, kidney or liver activities. Therefore the significant inhibition of neointimal hyperplasia in the POC-model after simultaneous intra/extravascular administration of $50 \mu \mathrm{g} / \mathrm{mL}$ of diltiazem has to be considered as an absolute minimum. It may be neccessary to increase both the period of administration and the concentration of diltiazem in order to reproduce an antiproliferative effect in vivo.

Although the domestic pig mimics the human situation very closely major differences endure between porcine and human tissue. Lately our laboratory has demonstrated [22] that the maximal proliferative response after ex vivo ballooning in human organ cultures of renal arteries (HOCs) occurred at day 21 after ballooning, followed by a maximum of neointimal thickening at day 56. These POC/HOC species differences with a maximum of antiproliferative activity at day $7 / 21$ and a maximum of neointimal hyperplasia at day 28/56 will be of extreme importance if the time period of simultaneous intra/extravascular administration of diltiazem in humans shall be estimated in the future.

The ex vivo POC-model [9] has the unique advantage that it can be compared easily to an in vivo model of thesame species, the domestic pig [21]. Both in the ex vivo POCmodel [9] and the in vivo domestic pig model [21] the maximal reactive cell proliferation and the maximal neointimal thickening after vascular injury occures at thesame days: day 7 respectively day 28 . It might be speculated that the similarities between the data of the ex vivo POCmodel and the in vivo domestic pig model can be discovered as well between the ex vivo HOC-model and the situation of the clinical patient after angioplasty. The unknown in vivo situation of the clinical patient might therefore be calculated or at least approached by the three understood variables. If that hypothesis is confirmed by 
other groups and by our own future research the combination of the in vivo domestic pig model with the ex vivo POC- and HOC-models may offer the closest approach to human coronary artery restenosis so far described.

The ex vivo/in vivo analogous conclusion can be applied to approach the reaction of human coronary arteries in the clinical patient after simultaneous intra/extravascular administration of high dose diltiazem. Data of the study presented would correspond to variable 1 , data of variable 2 and 3 are not yet available. Therefore the following studies should be carried out in the near future: 1) simultaneous intra/extravascular administration of diltiazem in the domestic pig for a period of 7 days, detection of reactive cell proliferation and neointimal thickening at day 7 and day 28, and 2) simultaneous intra/extravascular administration of diltiazem in the ex vivo HOC-model for a period of 21 days and detection of reactive cell proliferation and neointimal thickening at day 21 and day 56 .

Analogous conclusions based on the relation between ex vivo/in vivo domestic pig models and the ex vivo HOCmodel have the potential to abridge porcine/human species differences. Due to the significant importance of this hypothesis we would like to encourage other groups in the field to focus human restenosis by applying ex vivo POC[9] and HOC-models [22] in combination with the in vivo domestic pig model [21].

\section{References}

I. Holmes DR, Vlietstra RE, Smith HC, et al: Restenosis after percutaneous transluminal coronary angioplasty (PTCA): a report from the PTCA Registry of the National Heart, Lung, and Blood Institute. Am J Cardiol. 1984, 53:77C-8IC

2. Ferns GA, Avades TY: The mechanisms of coronary restenosis: insights from experimental models. Int J Exp Pathol. 2000, 81:6388

3. Voisard R, Seitzer U, Baur R, Dartsch PC, Osterhues H, Höher M, Hombach $V$ : A prescreening system for potential antiproliferative agents: implications for local treatment strategies of postangioplasty restenosis. Int J Cardiol I995, 5 I:I 1 -28

4. Voisard R, Kucharczyk E, Baur R, Deininger U, Hombach V: Simultaneous intra/extraluminal drug administration as new approach for the treatment of reactive smooth muscle cell proliferation after vascular injury: Use of the pericardium as additional drug depot? Eur Heart $\int 2000,2$ I:PI550

5. Verrier RL, Waxman S, Lovett EG, Moreno R: Transatrial access to the normal pericardial space: a novel approach for diagnostic sampling, pericardiocentesis, and therapeutic interventions. Circulation 1998, 98:2331-2333

6. Laham RJ, Simons M, Hung D: Subxyphpoid access of the normal pericardium: a novel delivery technique. Catheter Cardiovasc Interv 1999, 47:109-III

7. Macris MP, Igo SR: Minimally invasive access of the normal pericardium: initial clinical experience with a novel device. Clin Cardiol 1999, 22:136-9

8. Schwartz RS, Murphy JG, Edwards WD, Camrud AR, Vlietstra RE, Holmes DR Jr: Restenosis after balloon angioplasty: a practical proliferative model in porcine coronary arteries. Circulation 1990, 82:2190-2200

9. Voisard R, Jensch V, Baur R, Höher M, Hombach V: A coronary porcine organ culture system for studies of postangioplasty cell proliferation. Coron Artery Dis 1995, 6:657-665

10. Voisard R, Koschnick S, Baur R, Vogel U, Mattfeldt T, Hemmer W, Hannekum A, Höher M, Hombach V: High dose diltiazem pre- vents migration and proliferation of vascular smooth muscle cells in various in-vitro models of human coronary restenosis. Coron Artery Dis 1997, 8:189-201

II. Sachs L: Angewandte Statistik 1984230-235

12. Ciezki JP, Hafeli UO, Song P, Urankar-Nagy N, Ratliff NB, Rybicki L, Brill K, Meier D: Parenchymal cell proliferation in coronary arteries after percutaneous transluminal coronary angioplasty: a human tissue bank study. Int J Radiat Oncol Biol Phys 1999, I:45:963-968

13. Simon AD, Giedd KN, Schwartz A, Wang A, Sun J, Chen HJ, Marboe C, Stein CA, Rabbiani LE: Porous balloon delivery of S-dC28 does not prevent restenosis in the porcine coronary artery model of balloon injury. Antisense Nucleic Acid Drug Dev 1999, 9:549-553

14. Corcos T, David PR, Val PG, Renkin J, Dangoisse V, Rapold HG, et al: Failure of diltiazem to prevent restenosis after percutaneous transluminal coronary angioplasty. Am Heart J 1985, 109:926931

15. O'Keefe JH, Giorgi LV, Hartzler GO, Good TH, Ligon RW, Webb DL, et al: Effects of diltiazem on complications and restenosis after coronary angioplasty. Am J Cardiol I991, 67:373-376

16. Unverdorben M, Kunkel B, Leucht M, Bachmann K: Reduction of restenosis after PTCA by diltiazem? Circulation 1992, 86:153

17. Buckley MMT, Grant SM, Goa KL, McTavish D, Sorkin EM: Diltiazem: a reappraisal of its pharmacological properties and therapeutic use. Drugs 1990, 39:757-806

18. Koo EWY, Gotlieb Al: Neointimal formation in the porcine aortic organ culture: cellular dynamics over I month. Lab Invest 199|, 64:743-753

19. Kocher O, Skalli O, Bloom WS, Gabbiani G: Cytoskeleton of rat aortic smooth muscle cells: normal conditions and experimental intimal thickening. Lab lnvest 1984, 50:645-652

20. Voisard R, Fischer R, Oßwald M, Voglic S, Baur R, Susa M, Koenig W, Hombach V: Aspirin (5 mM) inhibits key events of early atherosclerosis and restenosis in a 3D human coronary in vitro model. 200I. Circulation 200I, 103:1688-1694

21. Carter AJ, Laird JR, Farb A, Kufs W, Wortham DC, Virmani R: Morphologic characteristics of lesion formation and time course of smooth muscle cell proliferation in a porcine proliferative restenosis model. J Am Coll Cardiol 1994, 24: I398-I 405

22. Voisard R, v. Eicken J, Baur R, Gschwend J, Wenderoth U, Kleinschmidt K, Hombach V, Höher M: A human arterial organ culture model of postangioplasty restenosis: results up to $\mathbf{5 6}$ days after ballooning. Atherosclerosis 1999, 144:123-134

Publish with BioMed Central and every scientist can read your work free of charge

"BioMedcentral will be the most significant development for disseminating the results of biomedical research in our lifetime." Paul Nurse, Director-General, Imperial Cancer Research Fund

Publish with BMC and your research papers will be:

- available free of charge to the entire biomedical community

- peer reviewed and published immediately upon acceptance

- cited in PubMed and archived on PubMed Central

- yours - you keep the copyright

Submit your manuscript here:

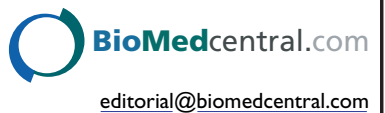

